\title{
Nodule-Forming Pseudoangiomatous Stromal Hyperplasia of the Breast: Report of Three Cases
}

Canan Kelten ${ }^{1}$, Ceren Boyaci ${ }^{1}$, Cem Leblebici ${ }^{1}$, Mehmet Ali Nazli², Şefika Aksoy ${ }^{3}$, Didem Can Trabulus ${ }^{3}$, Erol Rüştü Bozkurt ${ }^{1}$ ${ }^{1}$ Department of Pathology, İstanbul Research and Training Hospital, İstanbul, Turkey

${ }^{2}$ Department of Radiology, İstanbul Research and Training Hospital, İstanbul, Turkey

${ }^{3}$ Department of General Surgery, İstanbul Research and Training Hospital, İstanbul, Turkey

\begin{abstract}
Pseudoangiomatous stromal hyperplasia (PASH) is a benign proliferative entity of mammary stroma. It is generally found as an incidental finding. It may rarely present as a palpable nodule. Three patients, who were 29, 45, and 58 years of age, were referred to our clinic with nodule and pain in the breast. The physical examinations and ultrasound findings of all three patients were consistent with fibroadenoma. Core biopsies were performed and reported as "benign breast parenchyma including stromal fibrosis." PASH areas were noted in one case. The excision specimens were observed as solid nodular masses with smooth external surfaces and white in colour. Microscopically, well-demarcated hyalinized stroma, including slit-like pseudovascular spaces lined by bland spindle cells, was observed. Immunohistochemically, these cells showed positive staining for CD34 and negative staining for CD31. Nodule-forming PASH mostly confuses with fibroadenoma with respect to clinical examination and radiological findings. Definite diagnosis requires histopathological verification. Differential diagnosis should be made with low grade angiosarcoma, fibroepithelial tumors, and myofibroblastoma.
\end{abstract}

Keywords: Breast, nodule, pseudoangiomatous stromal hyperplasia

\section{Introduction}

Pseudoangiomatous stromal hyperplasia (PASH) is a benign proliferative entity of mammary stroma. It is characterized by slit-like pseudovascular spaces lined by myofibroblasts in a hyaline stroma $(1,2)$. We mostly characterize PASH as an incidental finding accompanying several benign or malignant lesions of breast while evaluating the surgical excision specimens microscopically. Occasionally, it may comprise a mass and therefore present as a palpable nodule clinically (3). It has been reported as either a rapidly enlarging nodule suspicious of malignancy or direct transformation to malignancy in single case reports $(4,5)$. Here, we report three nodular PASH cases.

\section{Case Presentation}

\section{Case 1}

A 29-year-old Caucasian woman was referred to our clinic with mastodynia for 1.5 months. No history was noted for oral contraceptive use. Physical examination revealed a mobile and well-defined mass in the mid-outer quadrant in her right breast, consistent with fibroadenoma. Similarly, ultrasound findings showed a deeply located, hypoechoic, homogeneous solid nodule with well-defined borders (25 $\times 10 \mathrm{~mm}$ ) (Figure 1a). Core needle biopsy was performed and reported as a "columnar cell lesion without atypia, fibrocystic changes in the form of fibrosis, dilated ducts, and extensive PASH areas completely involving one of the tissue fragments". The lesion was excised. Macroscopically, the specimen was a rubbery, well-circumscribed nodular mass with a diameter of $2 \mathrm{~cm}$. The cut surface was solid in consistency and white in colour. No hemorrhage, necrosis, or leaf-like pattern was described. Microscopically, a well-demarcated, extensively hyalinized stroma, including randomly distributed lobular units and a few dilated ducts, was observed. Some of the lobular units showed columnar cell changes without atypia. In addition, microcalcification associated with benign ducts and acinus within the lesion was noted. Both intralobular and interlobular hyalinized stroma displayed widespread, slit-like pseudovascular spaces lined by bland spindle cells (Figure 1b). No nuclear atypia or mitosis was detected in the cells lining the slit-like spaces. Immunohistochemically, these cells showed positive staining for $\mathrm{CD} 34$, smooth muscle actin, focal positivity for progesterone receptors, and negative staining for estrogen receptors and CD31. The specimen was reported as "Nodular PASH," with a brief comment that emphasized the benign nature of the lesion and possibility for local recurrence. No recurrence was reported after 34 months following excision.

This study was presented at the $23^{\text {th }}$ National Pathology Congress and $26^{\text {th }}$ European Congress of Pathology. 

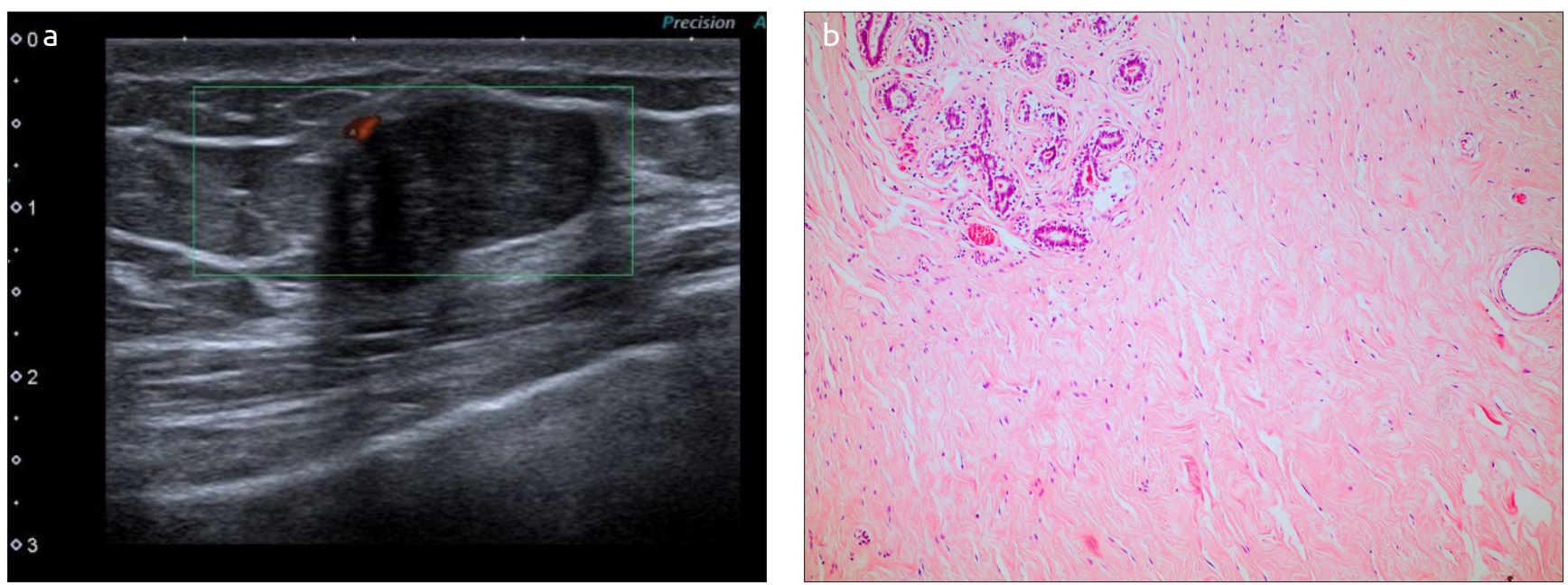

Figure 1. a, b. (a) Homogeneous solid nodule with well-defined borders (b) Pseudovascular spaces lined by bland spindle cells in intralobular and interlobular stroma. ( $\times 200 \mathrm{H} \& \mathrm{E})$
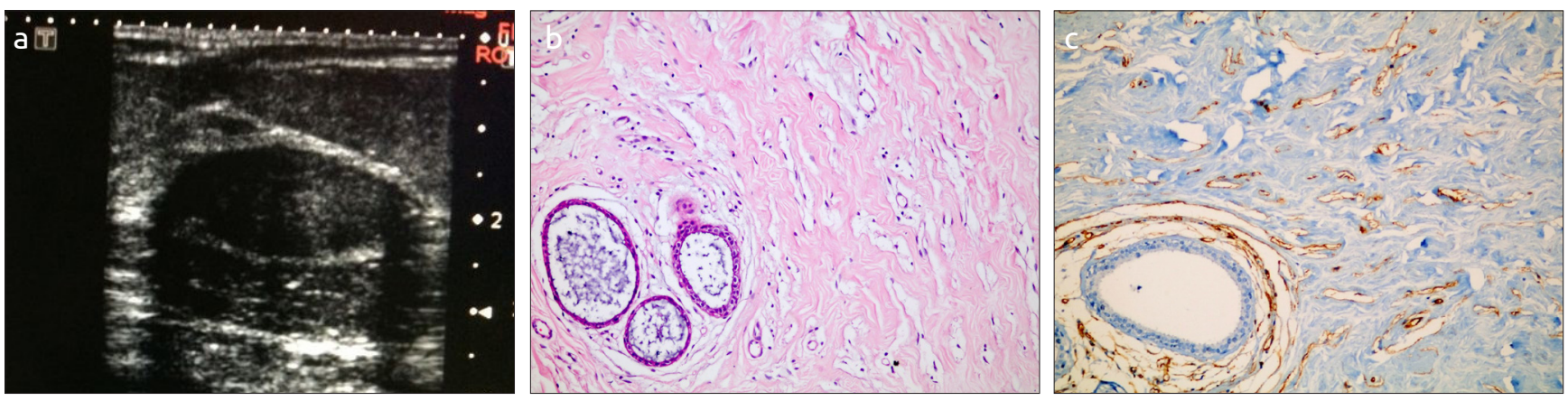

Figure 2. a-c. (a)Homogeneous, hypoechoic, solid nodule with well-defined borders parallel to the pectoral muscle (b) Slit-like spaces lined by bland spindle cells with no nuclear atypia or mitosis ( $\times 400 \mathrm{H \& E})(\mathrm{c})$ CD34 positivity in spindle cells lining pseudovascular spaces $(\times 400)$

\section{Case 2}

A 58-year-old Caucasian postmenopausal woman was referred to our clinic with mastodynia for a while. No history was noted for oral contraceptive use or hormone replacement therapy. Physical examination revealed a mobile and well-defined mass in the upper quadrant of her right breast. No calcification was detected mammographically. Ultrasound findings showed a homogeneous, hypoechoic, solid nodule (26 x $12.5 \mathrm{~mm}$ ), with well-defined borders parallel to the pectoral muscle (Figure 2a). No posterior enhancement was noted. Core needle biopsy was performed and reported as "fibrocystic changes composed of stromal fibrosis, dilated ducts and apocrine metaplasia, and focally PASH areas in the stroma”. The lesion was excised with a cuff of normal breast tissue. Macroscopically, the excision specimen was a well-circumscribed nodular mass $(2.5 \times 1.5 \times 1.5 \mathrm{~cm})$ with a solid cut surface. Microscopically, a few benign ducts and lobules were observed in a hyalinized stroma. The stroma displayed multifocal, slit-like pseudovascular spaces lined by bland spindle cells with no nuclear atypia or mitosis (Figure 2b). Immunohistochemically, the cells lining the slitlike spaces showed positivity for CD34 (Figure 2c) and negativity for CD31. Focally, weak positive staining for estrogen and progesterone receptors was noted. The specimen was reported as "Nodular PASH." No recurrence was reported after 23 months following excision.

\section{Case 3}

A 45-year-old Caucasian woman was referred to our clinic with a mass in her left breast for a long time (exact time unknown). No history was noted for oral contraceptive use. Physical examination revealed a nonfixed and well-defined mass in the upper-outer quadrant, consistent with fibroadenoma. Mammography showed an ovoid, well-defined mass of high density with no calcification, suggestive of a benign lesion (Figure 3a). Ultrasound findings revealed a hypoechoic, solid nodule $(39 \times 15 \mathrm{~mm})$ with well-defined borders. The lesion was beneath the skin, and its long axis was parallel to pectoral muscle. Core needle biopsy was performed and reported as "extensive stromal hyalinization including multifocal PASH areas and intraductal papilloma”. The lesion was excised with a cuff of normal breast tissue. Macroscopically, the excision specimen was a rubbery, well-circumscribed nodular mass with a diameter of $4 \times 3 \times 2.5 \mathrm{~cm}$ (Figure 3b). Microscopically, there were lobular units and a few dilated ducts with apocrine metaplasia in a well-demarcated hyalinized stroma. Minimal dilatation was observed in some lobular acinus and ducts. Periductal and perilobular stroma displayed multifocal, slit-like pseudovascular spaces lined by bland spindle cells with no nuclear atypia or mitosis (Figure 3c). Immunohistochemically, the cells lining the slit-like spaces showed positivity for CD34 and negativity for CD31. There was no staining for estrogen and progesterone receptors. The specimen was reported as "Nodular PASH." No recurrence was reported after 14 months following excision.

\section{Discussion and Conclusions}

The pathogenesis of PASH is still controversial. However, clinicopathological findings support that PASH is a benign lesion of mammary stroma growing dependent of hormonal status. For example, patients with PASH have been mostly described in premenopausal women and/or in women with a history of oral contraceptive use or hormone 

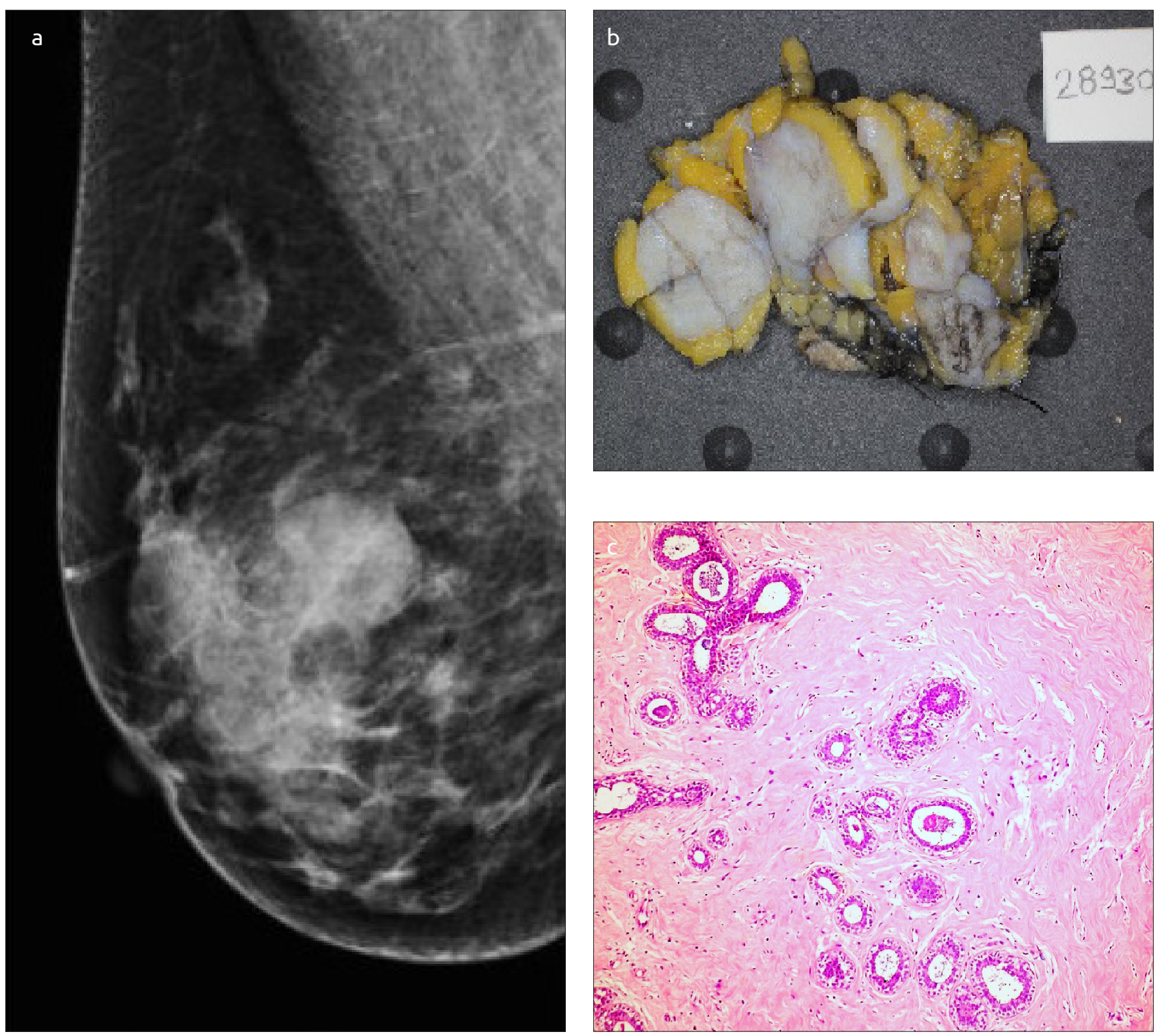

Figure 3. a-c. (a) Ovoid, well-defined mass of high density with no calcification (b) Rubbery, well-circumscribed solid, nodular mass, white in colour (c) Minimally dilated acinar structures surrounded with PASH $(\times 200 \mathrm{H} \& \mathrm{E})$

replacement treatment (6). PASH has also been described in men with gynecomastia and a trans-sexual patient taking exogenous hormones $(7,8)$. Furthermore, immuohistochemical positivity for the progesterone hormone receptor has been observed in stromal cells (6). Two of three patients were premenopausal in this report and showed immunopositivity for the progesterone receptor. However, no history of oral contraceptive use or hormone replacement treatment was obtained in the postmenopausal patient.

PASH areas generally have been identified as small, microscopic stromal foci in the background of several breast lesions. These lesions are observed in fibrocystic changes, fibroepithelial lesions (phylloides tumor and fibroadenoma), stromal alterations surrounding columnar cell changes, and even in invasive breast carcinoma (2). Similarly, we described columnar cell changes in one case (Case 1) and fibrocystic changes in all cases. Nodule-forming PASH mostly confuse with fibroadenoma with respect to the clinical examination and radiological findings as in our cases. It has been clinically described as a non- hypoechoic mass that is generally without calcification has been defined in ultrasonographic examination (9). If the core needle biopsy from a nodular lesion contains a large amount of PASH areas, then the possibility of nodular PASH may come to mind. Therefore, the exact diagnosis requires a histopathological examination of the entire biopsy material. Because one of our cases (Case 1) showed extensive PASH areas without a background of fibroepithelial lesion in core needle biopsy material, we could mention the possibility for nodular PASH in the comment of the pathology report based on the radiologic findings.

The histopathological differential diagnosis of PASH includes benign and malignant lesions of the breast, such as myofibroblastoma and low grade angiosarcoma $(3,10)$. Myofibroblastoma is also a well-circumscribed, slowly growing, nodular lesion that is generally located in the subareolar region. Both physical examination and radiologic findings of myofibroblastoma generally suggest fibroadenoma. Microscopically, short intersecting fascicles of bland spindle cells within collagen fibers were observed in myofibroblastoma. While the entrapment of benign breast ducts and lobules is rare in myofibroblastoma, it is generally 
noted in nodular PASH. Similarly, all of our cases displayed a varying amount of benign breast ducts and lobules in the nodular stroma of PASH. However, immunostainings do not help to distinguish nodular pash from myofibroblastoma since the origin of cells is same in both lesion. Angiosarcoma is an important neoplasm that must be differentiated from PASH. Unlike nodule-forming PASH, angiosarcoma is a hemorrhagic mass with irregular margins because of infiltrative vascular channels lined by malignant endothelial cells showing nuclear atypia and mitotic activity. Because these channels are true vascular composition, they show positive immunostaining for vascular markers such as CD31 and Factor VIII. However, the spindle cells originated from the myofibroblast in $\mathrm{PASH}$, and they show positivity for vimentin and CD34 but negativity for vascular markers. All of our cases showed negative staining for CD31 and positive staining for CD34, consistent with myofibroblastic cell origin. Occasionally, multinucleated stromal giant cells lining the pseudovascular spaces have been reported in nodular PASH, with a clinical history of rapidly growing breast mass (11). The myoepithelial cell origin (CD34 positivity, Factor VIII negativity) is mostly considered for atypical stromal giant cells. The atypical stromal cells have also been reported in the fibroepithelial tumors (12). We did not notice atypical stromal cells in all the cases.

Prognosis of PASH is considered as good. The treatment involves the surgical excision of the nodular mass. Recurrences have been described in patients with possible incomplete surgical excision.

Consequently, PASH is a benign stromal lesion and generally defined as small microscopic foci in the surgical excision material. It is important to be aware of this lesion because the histopathological differential diagnosis includes both benign and malignant lesions of the breast. Based on clinical and radiological analysis, PASH may rarely present as a solid, well circumscribed, hypoechoic nodule, similar to fibroadenoma. The presence of extensive or diffuse PASH areas in a core needle biopsy should alert the pathologist with respect to the possibility for nodular PASH in a proper clinical setting. The exact treatment and diagnosis requires surgical excision of the entire mass.

Informed Consent: Written informed consent was obtained from patient who participated in this study.

Peer-review: Externally peer-reviewed.

Author Contributions: Concept - C.B., C.K.; Design - C.B., M.A.N., D.C.T.; Supervision - C.L., Ş.A., E.R.B.; Funding - M.A.N., E.R.B.; Data Collection and/or Processing - C.B., C.L., M.A.N.; Analysis and/or Interpretation - Ş.A.,
D.C.T.; Literature Review - C.K., Ş.A., D.C.T.; Writer - C.K., C.B.; Critical Review - C.L., E.R.B.

Conflict of Interest: No conflict of interest was declared by the authors.

Financial Disclosure: The authors declared that this study has received no financial support.

\section{References}

1. Vuitch MF, PP Rosen, RA Erlandson. Pseudoangiomatous hyperplasia of mammary stroma. Hum Pathol 1986; 17:185-191. (PMID: 3949338) [CrossRef]

2. Ibrahim RE, CG Sciotto, N Weidner. Pseudoangiomatous hyperplasia of mammary stroma. Some observations regarding its clinicopathologic spectrum. Cancer 1989; 63:1154-1160. (PMID: 2917318)

3. Drinka EK, Bargaje A, Erşahin ÇH, Patel P, Salhadar A, Sinacore J, Rajan P. Pseudoangiomatous stromal hyperplasia (PASH) of the breast: a clinicopathological study of 79 cases. Int J Surg Pathol 2012; 20:54-58. (PMID: 21862488) [CrossRef]

4. Salemis NS. Giant tumoural pseudoangiomatous stromal hyperplasia of the breast in the adolescence. ANZ J Surg 2011; 81:469-470. (PMID: 22295353) [CrossRef]

5. Nassar H, Elieff MP, Kronz JD, Argani P. Pseudoangiomatous stromal hyperplasia (PASH) of the breast with foci of morphologic malignancy: a case of PASH with malignant transformation? Int J Surg Pathol 2010; 18:564-569. (PMID: 18611932)

6. Anderson C, Ricci A Jr, Pedersen CA, Cartun RW. Immunocytochemical analysis of estrogen and progesterone receptors in benign stromal lesions of the breast. Evidence for hormonal etiology in pseudoangiomatous hyperplasia of mammary stroma. Am J Surg Pathol 1991; 15:145-149. (PMID: 1989462) [CrossRef]

7. Bowman E, Oprea G, Okoli J, Gundry K, Rizzo M, Gabram-Mendola S, Manne U, Smith G, Pambuccian S, Bumpers HL. Pseudoangiomatous stromal hyperplasia (PASH) of the breast: a series of 24 patients. Breast J 2012; 18:242-247. (PMID: 22583194) [CrossRef]

8. Badve S, Sloane JP. Pseudoangiomatous hyperplasia of male breast. Histopathology 1995; 26:463-466. (PMID: 7544764) [CrossRef]

9. Mercado CL, Naidrich SA, Hamele-Bena D, Fineberg SA, Buchbinder SS. Pseudoangiomatous stromal hyperplasia of the breast: sonographic features with histopathologic correlation. Breast J 2004; 10:427-432. (PMID: 15327497) [CrossRef]

10. Virk RK, Khan A. Pseudoangiomatous stromal hyperplasia: an overview. Arch Pathol Lab Med 2010; 134:1070-1074. (PMID: 20586640)

11. Comunoğlu N, Comunoğlu C, Ilvan S, Calay Z, Müslümanoğlu. Mammary pseudoangiomatous stromal hyperplasia composed of predominantly giant cells: an unusual variant. Breast J 2007; 13: 568-570. (PMID: 17983397) [CrossRef]

12. Hanna W, AL-Maghrabi J, Malik A. Aggressive giant fibroepithelial lesion with unusual vascular stroma-a case report. Mod Pathol 2003; 16:823827. (PMID: 12920228) [CrossRef] 